\title{
Heterochronic Detection Through a Function for the Ontogenetic Variation of Bone Shape
}

\author{
J. Cubo*†, D. Azagra \\ * Equipe Formations Squelettiques, CNRS UMR 8570-2, Pl. Jussieu-Case, 7077 - 75251, Paris Cedex \\ 05, France $\$$ Departamento de Analisis Matematico, Facultad de Ciencias Matematicas, Universidad \\ Complutense de Madrid, 28040 Madrid, Spain and \$Departament de Biologia Animal, Universitat de \\ Barcelona, Av. Diagonal, 645, 08028 Barcelona, Spain
}

\begin{abstract}
Heterochrony, evolutionary modifications in the rates and/or the timing of development, is widely recognized as an important agent of evolutionary change. In this paper, we are concerned with the detection of this evolutionary mechanism through the analysis of long bone growth. For this, we provide a function $\sigma(t)$ for the ontogenetic variation of bone shape by taking the ratio of two Gompertz curves explaining, respectively, the relative contribution to long bone growth of (a) endochondral ossification and (b) periosteal ossification. The significance of the fitting of this function to empirical data was tested in Anas platyrhynchos (Anseriformes). In this function $\sigma(t)$, the time $t_{m}$ at which periosteal growth rate first equalizes endochondral growth rate was taken as the timing parameter to be compared between taxa. On the other hand, the maximum rate of ontogenetic change in bone shape (maximum slope, $\beta$ ) from hatching to $t_{m}$ was taken as the rate parameter to be compared. Comparisons of these parameters between the plesiomorphic condition and the derived character state would provide evidence for hypomorphosis (earlier occurrence of $t_{m}$ ), hypermorphosis (delayed occurrence of $t_{m}$ ), deceleration (smaller $\beta$ ) or acceleration (higher $\beta$ ).

Regarding the phylogenetic context, the ancestral condition for the character of interest should be estimated to polarize the direction of the heterochronic change. We have quantified the influence of the phylogenetic history on the variation of adult bone shape in a sample of 13 species of Anseriformes and 17 species from other neornithine orders of birds by using permutational phylogenetic regressions. Phylogenetic effects are significant, and this fact allows the optimization of bone shape onto a phylogenetic tree of Anseriformes to estimate the ancestral condition for Anas platyrhynchos.
\end{abstract}

\section{Introduction}

The identification of the evolutionary processes underlying the generation of phylogenetic patterns is a major goal of comparative biology

$\dagger$ Author to whom correspondence should be addressed. E-mail: cubo@ccr.jussieu.fr
(Eldredge \& Cracraft 1980; Pagel, 1997, 1999). Heterochrony, evolutionary modifications in the rates and/or the timing of development, is widely recognized as an important agent of evolutionary change (Gould, 1977, 2000; Alberch et al., 1979). The detection of the incidence of this evolutionary process in the evolution of a clade 
requires both (A) a phylogenetic hypothesis and (B) the quantification of growth parameters in the different taxa (Klingenberg, 1998).

(A) Phylogenetic context: Ancestral character states should be estimated to polarize the direction of the heterochronic change (Boughton et al., 1991).

(B) Quantification of growth. Three variables are important in heterochronic detection: age, size (increase in either spatial dimensions or mass) and shape (a non-dimensional measurement of the proportions of an organ) (Godfrey \& Sutherland, 1995a, b, 1996).

In this paper, we are concerned with the detection of heterochrony through the analysis of long bone growth. For this, we use Anas platyrhynchos (Anseriformes) as an animal model. First, we estimate ancestral character states (regarding bone shape) for Anas platyrhynchos. Afterwards, we develop a function for the ontogenetic variation of bone shape by taking the ratio of two Gompertz curves that explain, respectively, the relative contribution to long bone growth of (a) endochondral ossification and (b) periosteal ossification. Then, we test the significance of the fitting of this function to empirical data in Anas platyrhynchos. Finally, we carry out a comparative analysis of these growth parameters.

\section{Phylogenetic Context}

As quoted above, the ancestral condition for the character of interest (bone shape) should be estimated to polarize the direction of the heterochronic change (Boughton et al., 1991). A number of methods have recently been proposed to estimate the ancestral character states for continuous characters (Pagel, 1999). But, prior to the use of these methods, we should verify that phylogenetic effects on the variation of bone shape are significant.

\section{QUANTIFICATION OF PHYLOGENETIC EFFECTS}

Bone shape diversity can result from the phylogenetic legacy of the clade. According to this hypothesis, closely related species have a recent last common ancestor, they share a greater portion of genotype and they might tend to share similar morphologies than distantly related species (Harvey \& Pagel, 1991). However, bone shape variation may also be the result of recent adaptive constraints independent of higher levels of phylogeny.

To quantify the influence of the phylogenetic history on the variation of bone shape in adults, we measured the ratio bone length (the result of endochondral ossification)/bone diameter (the result of periosteal ossification) in a sample of humeri and femora of 13 anseriform species and 17 species from other neornithine orders of birds (see Appendix C). For each pair of species, their morphological dissimilarity (regarding bone shape) is compared with their phylogenetic distance. To quantify the phylogenetic distances, we have used a consensual phylogeny of birds. Recent molecular (van Tuinen \& Hedges, 2001) and palaeontological (Cracraft, 2001) studies have reached similar conclusions regarding the relationships among major groups of modern birds (Neornithes) : Palaeognathae (ratites and tinamous) and Neognathae (all other modern birds) are sister-groups; within Neognathae, Galliformes and Anseriformes are each other's closest relative, and they are grouped in Galloanserae; Galloanserae and Neoaves (all other neognath birds) are sister-groups. Most phylogenetic relationships among and within different orders of Neoaves are unresolved. Regarding Anseriformes and Ratites, we have used trees modified, respectively, from McCracken et al., 1999 and van Tuinen \& Hedges, 2001. Both molecular clock data (van Tuinen \& Hedges, 2001) and the fossil record (Cracraft, 2001, and references herein) were used to estimate divergence times between avian clades. Both studies agree in that modern birds (Neornithes) arose and radiated prior to the Cretaceous-Tertiary extinction event. Divergence times used for the split between major groups of birds were: Palaeognathae-Neognathae: 118.6 MYA, Galloanserae-Neoaves : 104.2 MYA, GalliformesAnseriformes : 89.8 MYA, basal Neoaves:89.3 MYA (van Tuinen \& Hedges, 2001). In general, no divergence times are available for within order comparisons. In these cases, the first occurrence in the fossil record of each order (the geological age of the oldest fossil of each order, see Cracraft, 2001, and references herein) 
was tentatively used as the age of divergence between the different species of this order. Two matrices were constructed with the comparisons for each pair of species: the morphological dissimilarity matrix (regarding bone shape) and the phylogenetic distance matrix (divergence times). Phylogenetic effects were quantified by using permutational phylogenetic regressions (Legendre et al., 1994): the morphological dissimilarity matrix was regressed to the phylogenetic distance matrix and the significance of this regression was tested by using the Mantel test (Böhning-Gaese \& Oberrath, 1999).

Phylogenetic effects were significant: phylogeny explains $18.35 \%(p=0.001)$ of the variation of humerus shape and $20.69 \%(p=0.001)$ of the variation of femur shape. Regarding bone shape, closely related species are more similar than distantly related species, and this fact allows the optimization of these characters onto a phylogenetic tree and the estimation of ancestral character states.

\section{ESTIMATION OF ANCESTRAL CHARACTER STATES}

According to Boughton et al. (1991), to polarize the direction of the heterochronic change the ancestral condition for the character of interest should be estimated (in our case study, the ancestral femur shape for Anas platyrhynchos). For this, we have used squaredchange parsimony, a method that minimizes the sum of squared changes along the branches of the phylogenetic tree (Maddison, 1991). The information required to use this method is: (A) data for the phenotypic variation of the trait under analysis (bone shape) in species closely related to the species of interest (Anas platyrhynchos) and (B) a dichotomous tree with the cladistic relationships among these species (modified from McCracken et al., 1999). We have used femur shape of 11 Anseriformes (tips of the phylogenetic tree) to estimate ancestral values (internal nodes of the tree) through squaredchange parsimony (Fig. 1). Femora of the outgroups of the clade $\{[$ (Bucephala-Somateria) Tachyeres]Anas $\}$ are robuster than femora of the species of this clade. Next, we will develop a function which would allow to determine

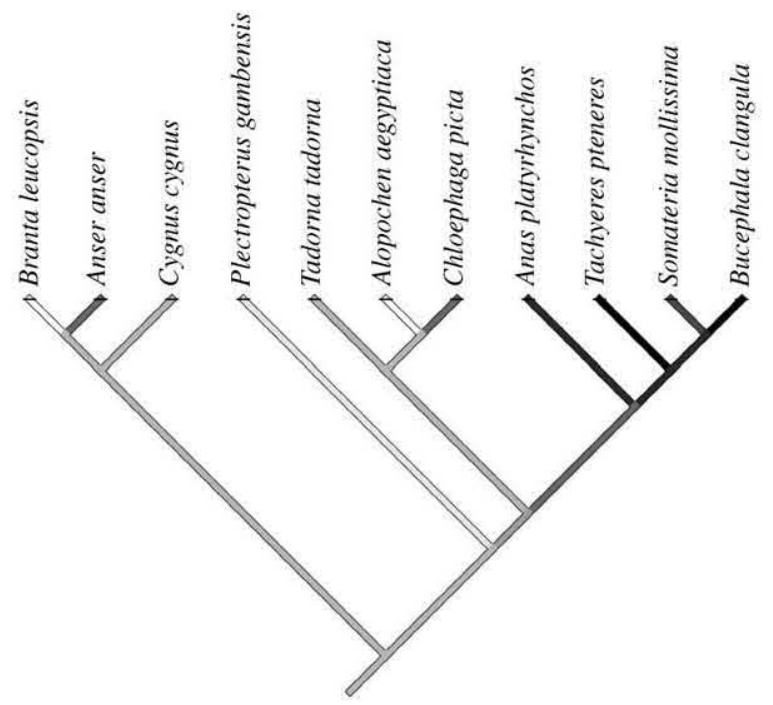

Frg. 1. Optimization of femur shape, the ratio bone length (the result of endochondral ossification)/bone diameter (the result of periosteal ossification), onto a phylogenetic tree of Anseriformes (modified from McCracken et al., 1999). The estimation of ancestral character states was carried out through squared-change parsimony, a method that minimizes the sum of squared changes along the branches (Maddison, 1991). ( $\square$ ) 8.741 -

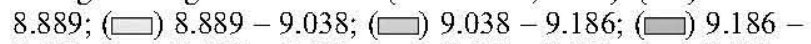

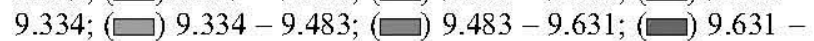
$9.779 ;$ ( $9.779-9.927$; $(\square) 9.927-10.076$; 10.078 -10.224 .

whether an heterochronic mechanism was involved in this morphological change.

\section{Quantification of Growth}

BONE SIZE

The first step to deduce a function for the variation of bone shape with age should be the search for a good descriptor of the ontogenetic variation of bone size. The Gompertz equation is recommended for modelling both body general growth and bone growth because it has provided the best fit to the empirical data in birds (Laird, 1965; Ricklefs, 1973; Ricklefs \& Marks, 1985) as well as in mammals (Maunz \& German, 1997; Fiorello \& German, 1997). The Gompertz law states that the growth of a structure is the outcome of the interaction of two opposing processes: (a) an initial exponential proliferation of the system and (b) an ulterior exponential decay of this primary exponential growth rate (Laird, 1965, 1966; Laird et al., 1965). In the case 
of long bones, endochondral ossification accounts for bone growth in length (Cubo et al., 2000 ), and it has been shown that bone growth rates in length decay exponentially with age (Cubo, 2000). On the other hand, periosteal ossification is responsible for bone growth in diameter (Castanet et al., 1996). In periosteal ossification, mitotic frequency also decays exponentially with age at mouse mid-diaphyseal femoral periosteum $\left(R^{2}=0.972, p<0.001, n=5\right.$; empirical data have been calculated with data taken from Tonna, 1961). The mathematical expression of the Gompertz law is as follows:

$$
y=w \exp \left[(l / k)\left(1-\mathrm{e}^{(-k t)}\right)\right],
$$

where $y$ is the size of the structure under analysis, $w$ is its initial size, $l$ is the initial growth rate, $k$ is the rate of exponential decay of the initial growth rate and $t$ is the time (Laird et al., 1965, Maunz \& German, 1997).

We have chosen the mallard (Anas platyrhynchos) as a model, on the basis that significant information is available on the growth dynamics of the appendicular bones of this species (Castanet et al., 1996). Unfortunately, such information is not available for closely related species of Anas platyrhynchos. Firstly, we will test the hypothesis that the Gompertz equation is a good descriptor of bone growth in the mallard. For this, we will check whether this equation explains a reasonable amount of variance of scatter plots of bone size vs. age.
To test our hypothesis, the Gompertz general equation should be tailored to bone growth:

$$
\begin{gathered}
L=w_{l} \exp \left[\left(l_{l} / k_{l}\right)\left(1-\mathrm{e}^{\left(-k_{l} t\right)}\right)\right], \\
D=w_{d} \exp \left[\left(l_{d} / k_{d}\right)\left(1-\mathrm{e}^{\left(-k_{d} t\right)}\right)\right],
\end{gathered}
$$

where $L$ is the bone length, $D$ is the bone diameter, $w_{l}$ and $w_{d}$ are the initial bone length and the initial bone diameter, $l_{l}$ and $l_{d}$ are the initial endochondral and periosteal growth rates and $k_{l}$ and $k_{d}$ are the rates of exponential decay of the initial endochondral and periosteal growth rates, respectively.

The length and the diameter of humerus, radius, carpometacarpus, femur, tibiotarsus and tarsometatarsus of 63 mallards were measured with a caliper ( $\mathrm{ROCH}$, France) to the nearest $0.01 \mathrm{~mm}$. These mallards ranged from 11 prehatching to 200 post-hatching days, and the used bones formed part of a pre-existing collection (see Castanet et al., 1996, for additional information). Bone length and bone diameter were regressed to age through eqns (2) and (3), respectively. The significance of the nonlinear regression coefficients was tested through the calculation of the $F$ value and the corresponding probability (Table 1). The regression coefficients were highly significant for all bones analysed in this study. These results support our hypothesis that the Gompertz law is a good descriptor of periosteal and endochondral bone growth in the mallard. See Figs 2 and 3 for a graphic

TABLE 1

Statistics of the nonlinear regressions of bone size (length and diameter) to age through the Gompertz equation. Abbreviations: $D$, diameter; $L$, length

\begin{tabular}{llllll}
\hline & & $n$ & $R^{2}$ & $F$ & $p$ \\
\hline Humerus & $\mathrm{L}$ & 46 & 0.896 & 385.95 & $p<0.001$ \\
& $\mathrm{D}$ & 49 & 0.858 & 284.00 & $p<0.001$ \\
Radius & $\mathrm{L}$ & 50 & 0.898 & 428.54 & $p<0.001$ \\
& $\mathrm{D}$ & 50 & 0.836 & 242.51 & $p<0.001$ \\
Carpometacarpus & $\mathrm{L}$ & 43 & 0.894 & 353.39 & $p<0.001$ \\
Femur & $\mathrm{D}$ & 45 & 0.884 & 324.15 & $p<0.001$ \\
& $\mathrm{~L}$ & 57 & 0.957 & 1276.18 & $p<0.001$ \\
Tibiotarsus & $\mathrm{D}$ & 57 & 0.932 & 775.92 & $p<0.001$ \\
\multirow{2}{*}{ Tarsometatarsus } & $\mathrm{L}$ & 60 & 0.963 & 1552.05 & $p<0.001$ \\
& $\mathrm{D}$ & 62 & 0.885 & 492.07 & $p<0.001$ \\
& $\mathrm{~L}$ & 63 & 0.963 & 1631.00 & $p<0.001$ \\
& $\mathrm{D}$ & 63 & 0.923 & 740.40 & $p<0.001$ \\
\hline
\end{tabular}


representation as an example of the ontogenetic variations of radius length and radius diameter, respectively.

\section{BONE SHAPE}

The mathematical expression of the variation of bone shape over time can be obtained by dividing the function that accounts for bone endochondral growth by the function that explains bone periosteal growth. Assuming the Gompertz function, a mathematical expression for the ontogenetic variation of bone shape $(\sigma)$ can be obtained by dividing eqn (2) by eqn (3):

$$
\begin{aligned}
\sigma= & \left(w_{l} / w_{d}\right) \exp \left\{\left(l_{l} / k_{l}\right)\left(1-\mathrm{e}^{\left(-k_{l} t\right)}\right)\right. \\
& \left.-\left[\left(l_{d} / k_{d}\right)\left(1-\mathrm{e}^{\left(-k_{d} t\right)}\right)\right]\right\} .
\end{aligned}
$$

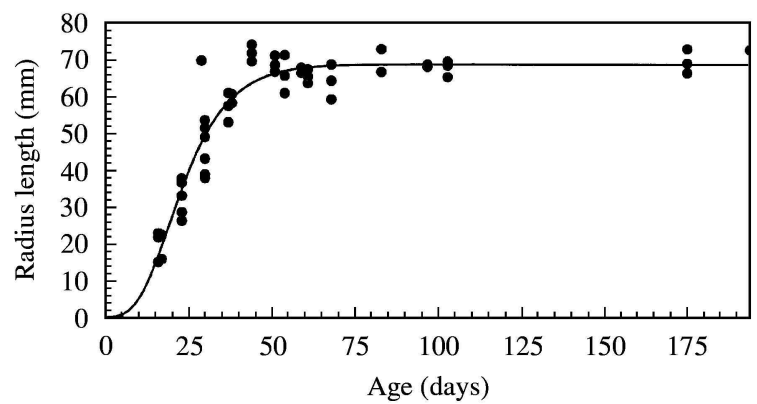

FIG. 2. Graphic representation of the ontogenetic variation of radius length in mallards. The scatter plot radius length vs. age has been fitted with the Gompertz function: $L=0.046 \exp \left[(0.755 / 0.103)\left(1-\mathrm{e}^{(-0.103 t)}\right)\right], n=50$, $R^{2}=0.898, F=428.54, p<0.001$, where $L$ is bone length and $t$ is time.

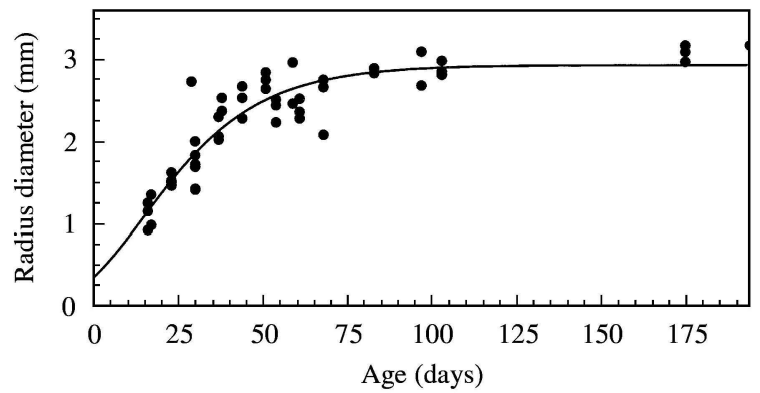

FIG. 3. Graphic representation of the ontogenetic variation of radius diameter in mallards. The scatter plot radius diameter vs. age has been fitted with the Gompertz function: $D=0.351 \exp \left[(0.109 / 0.051)\left(1-\mathrm{e}^{(-0.051 t)}\right)\right], \quad n=50$, $R^{2}=0.836, F=242.51, p<0.001$, where $D$ is the bone diameter and $t$ is the time.
Operating

$$
\begin{aligned}
\sigma= & \left(w_{l} / w_{d}\right) \exp \left\{\left[l_{l} / k_{l}\right]-\left[\left(l_{l} / k_{l}\right) \mathrm{e}^{\left(-k_{l} t\right)}\right]\right. \\
& \left.-\left[l_{d} / k_{d}\right]+\left[\left(l_{d} / k_{d}\right) \mathrm{e}^{\left(-k_{l} t\right)}\right]\right\} .
\end{aligned}
$$

Regrouping exponential terms

$$
\begin{aligned}
\sigma= & \left(w_{l} / w_{d}\right) \exp \left\{\left[l_{l} / k_{l}\right]-\left[l_{d} / k_{d}\right]\right\} \\
& \exp \left\{\left[\left(l_{d} / k_{d}\right) \mathrm{e}^{\left(-k_{l} t\right)}\right]-\left[\left(l_{l} / k_{l}\right) \mathrm{e}^{\left(-k_{l} t\right)}\right]\right\} .
\end{aligned}
$$

We shall denote

$$
a=\left(w_{l} / w_{d}\right) \exp \left\{\left[l_{l} / k_{l}\right]-\left[l_{d} / k_{d}\right]\right\}
$$

for short. We have then

$\sigma=a \exp \left\{\left[\left(l_{d} / k_{d}\right) \mathrm{e}^{\left(-k_{d} t\right)}\right]-\left[\left(l_{l} / k_{l}\right) \mathrm{e}^{\left(-k_{l} t\right)}\right]\right\}$,

where " $a$ " is a constant.

The next step will be to test whether eqn (8) is a good descriptor of the variation of bone shape with age in our animal model (Anas platyrhynchos). For this, nonlinear regressions between bone shape and age were carried out through eqn (8) (see Fig. 4 for a graphic representation of the ontogenetic variation of radius shape). The regression coefficients were highly significant for all the bones studied in mallards (Table 2) and, therefore, these results validate eqn (8) as a good descriptor of the ontogenetic variation of bone shape. In general, it could be stated that our function holds for the development of those bones whose growth is explained by the Gompertz function.

\section{Heterochronic Detection}

As it has been shown above, $\sigma(t)$ [eqn (8)] is an accurate tool to quantify the ontogenetic variation of bone shape. Since heterochrony involves evolutionary changes in timing and/or rates of development, the use of the function $\sigma(t)$ in heterochronic detection requires the definition of timing and rate parameters on it.

\section{TIMING PARAMETER}

According to Klingenberg (1998), evolutionary modifications in ontogeny can affect the size, shape and age at which the organism attains any particular developmental stage. We used the 


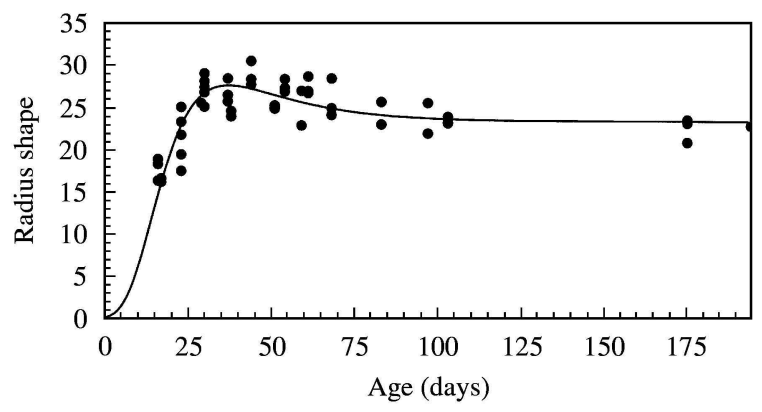

FIG. 4. Graphic representation of the ontogenetic variation of radius shape, measured as the ratio length/ diameter, in mallards. The scatter plot radius shape vs. age has been fitted with eqn (8): $\sigma(t)=23.587$ ex$\mathrm{p}\left[\left(2.137 \mathrm{e}^{-0.051 t}\right)-\left(7.330 \mathrm{e}^{-0.103 t}\right)\right], \quad n=50, \quad R^{2}=0.725$, $F=127.49, p<0.001$, where $\sigma$ is the bone shape and $t$ is the time.

TABLE 2

Statistics of the nonlinear regressions of bone shape (measured as the ratio bone length/bone diameter) to age through eqn (8), $\sigma(t)$

\begin{tabular}{lllll}
\hline & $n$ & $R^{2}$ & $F$ & $p$ \\
\hline Humerus & 46 & 0.602 & 66.75 & $p<0.001$ \\
Radius & 50 & 0.725 & 127.49 & $p<0.001$ \\
Carpometacarpus & 43 & 0.695 & 93.73 & $p<0.001$ \\
Femur & 57 & 0.416 & 40.02 & $p<0.001$ \\
Tibiotarsus & 59 & 0.714 & 143.00 & $p<0.001$ \\
Tarsometatarsus & 63 & 0.615 & 98.51 & $p<0.001$ \\
\hline
\end{tabular}

shape at which periosteal growth rate first equalizes endochondral growth rate as the developmental stage to be compared between the plesiomorphic and apomorphic conditions. The mathematical definition of this discrete stage is the time at which the slope of the function $\sigma(t)$ is zero (that means, the time at which the function has either a maximum or a minimum, $\left.t_{m}\right)$. See Appendix A for a deduction of the timing parameter $t_{m}$.

Figure 5 shows a graphic representation of the time $\left(t_{m}\right)$ at which the function $\sigma(t)$ attains its maximum in the case of the radius of mallards. This time corresponds to the shape $(M)$ at which periosteal growth rate first equalizes endochondral growth rate. Comparisons between the plesiomorphic time and the derived time at this ontogenetic stage $\left(t_{m}\right)$ would provide evidence for timing heterochrony. The cases of apomorphic $t_{m}$ significantly lower than plesiomorphic $t_{m}$ would be the evidence for hypomorphosis (terminology from Reilly et al.,

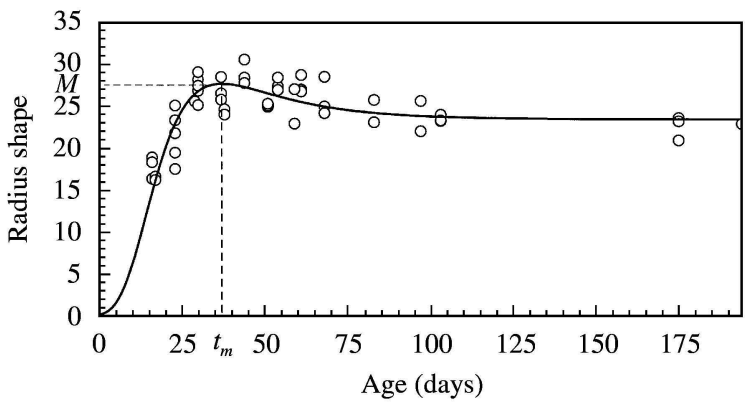

FIG. 5. Graphic representation of the time $\left(t_{m}\right)$ at which the function $\sigma(t)$ for the ontogenetic variation of bone shape attains its maximum $(M)$ in the radius of mallard. The mathematical definition of this discrete stage is the time $\left(t_{m}\right)$ at which the slope of the function $\sigma(t)$ is zero. Therefore, to calculate the parameter $t_{m}$, we shall consider the derivative of $\sigma(t)$ and calculate its zeros: $\sigma^{\prime}(t)=\sigma(t)$ $\left[0.755 \mathrm{e}^{(-0.103 t)}-0.109 \mathrm{e}^{(-0.051 t)}\right]$. The unique zero of $\sigma^{\prime}(t)$ is: $t_{m}=\left[\log \left(I_{d} / I_{l}\right)\right] /\left(k_{d}-k_{l}\right)=37.219$. At this time, the function $\sigma(t)$ for the ontogenetic variation of bone shape has its maximum: $M=\sigma\left(t_{m}\right)=27.715$.

1997). On the contrary, the cases of apomorphic $t_{m}$ significantly higher than plesiomorphic $t_{m}$ would be the evidence for hypermorphosis.

\section{RATE PARAMETER}

The maximum rate of ontogenetic change in shape will be calculated as the maximum slope $(\beta)$ of the function $\sigma(t)$ in the period that spans from hatching $\left(t_{0}\right)$ to the time at which the function attains its maximum $\left(t_{m}\right)$. See Appendix $\mathrm{B}$ for a deduction of the rate parameter $\beta$.

Figure 6 shows the calculation of the maximum slope $\beta$ in the radius of mallard. Comparisons of the plesiomorphic and the derived maximum rate of ontogenetic change in shape $(\beta)$ would provide evidence for rate heterochrony. The cases of apomorphic $\beta$ smaller than plesiomorphic $\beta$ would be the evidence for deceleration (terminology from Reilly et al., 1997). On the contrary, the cases of apomorphic $\beta$ higher than plesiomorphic $\beta$ would be the evidence for acceleration.

\section{Discussion}

COMPARATIVE ANALYSIS OF GROWTH PARAMETERS IN A PHYLOGENETIC CONTEXT

As quoted above, adult femora of the outgroups of the clade $\{[$ (Bucephala-Somateria) 


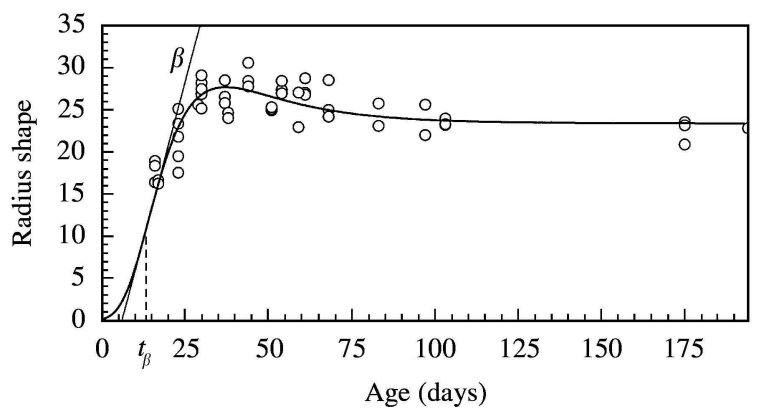

FIG. 6. Graphic representation of the maximum slope $(\beta$, the maximum rate of ontogenetic change in shape ) of the function $\sigma(t)$ in the period that spans from hatching $\left(t_{0}\right)$ to the time at which the function attains its maximum $\left(t_{m}\right)$ in mallards. In order to calculate the time $t_{\beta}$ at which the maximum slope $\beta$ is attained, we must find the first zero of the second derivative of $\sigma(t): \quad \sigma^{\prime \prime}(t)=\sigma(t)$ $\left[\left(0.570 e^{-0.206 t}\right)+\left(0.012 \mathrm{e}^{-0.102 t}\right)-\left(0.165 \mathrm{e}^{-0.154 t}\right)+\right.$ $\left.\left(0.006 e^{-0.051 t}\right)-\left(0.078 \mathrm{e}^{-0.103 t}\right)\right]$. The first zero of the second derivative $\sigma^{\prime \prime}(t)$ can be approximated by using any standard computer program: $t_{\beta}=13.998$. Then, we can find the maximum slope $\beta$ by substituting $t=t_{\beta}$ in the expression for $\sigma^{\prime}(t): \beta=\sigma^{\prime}\left(t_{\beta}\right)=\sigma^{\prime}(13.998)=1.489$. The equation of the tangent line to the graph of the function $\sigma(t)$ at the time $t_{\beta}$ is of the form: $y=\beta\left(t-t_{\beta}\right)+b$, where $b$ is the value of $\sigma(t)$ at $t_{\beta}$, that is, $b=\sigma\left(t_{\beta}\right)$.

Tachyeres]Anas\} are robuster than femora of the species of this clade. According to Boughton et al. (1991), the ancestral condition for the character of interest should be estimated to determine the direction of the heterochronic change. The optimization of femur shape onto the phylogenetic tree of Anseriformes (modified from McCracken et al., 1999) shows that, as expected, the femur of the last common ancestor of Anas platyrhynchos and Bucephala clangula is also robuster than the femur of Anas platyrhynchos (Fig. 1). Either an apomorphic $t_{m}$ significantly higher than plesiomorphic $t_{m}$ (hypermorphosis) or an apomorphic $\beta$ significantly higher than plesiomorphic $\beta$ (acceleration) would explain the derived character state found in Anas platyrhynchos. Future quantification on the growth parameters of the function developed in this paper $\left(t_{m}\right.$ and $\left.\beta\right)$ in species closely related to Anas platyrhynchos and the estimation of ancestral states would allow to test these hypotheses.

Related questions to this problem are the following. Considering that heterochrony often leads to a whole array of derived, but otherwise unrelated, character states (Cubo \&
Arthur, 2001), do particular developmental changes in other parts of the body of Anas platyrhynchos co-occur with femoral peramorphosis? In other words, is femoral peramorphosis linked by correlated development with peramorphosis in other structures of Anas platyrhynchos?

Finally, it has been shown that bone cortical thickness is an important parameter from the point of view of natural selection (Currey \& Alexander, 1985; Cubo \& Casinos, 2000). Both periosteal ossification and endosteal resorption/ endosteal ossification determine bone cortical thickness (Ricqles et al., 2000). The development of a mathematical function for the ontogenetic variation of bone cortical thickness would be an useful tool to complete the analysis of the evolution of bone growth.

We thank J. Belliure (Universite Paris VI, France) for collaborating in the edition of the figures and M. Laurin (CNRS, France) for allowing the use of MacClade 4.0. We gratefully acknowledge J. Y. Sire and two anonymous reviewers for insightful comments. This research was partially funded by grant PB95-0113-CO2-02 of the Spanish Gouvernment. J. Cubo had a post-doctoral grant from the "Ministerio de Educacion y Cultura" of the Spanish Government.

\section{REFERENCES}

Alberch, P., Gould, S. J., Oster, G. \& Wake, D. (1979). Size and shape in ontogeny and phylogeny. Paleobiology 5, 296-317.

Böhning-Gaese, K. \& Oberrath, R. (1999). Phylogenetic effects on morphological, life-history, behavioural and ecological traits in birds. Evol. Ecol. Res. 1, 347-364.

Boughton, D. A., Collette, B. B. \& McCune, A. R. (1991). Heterochrony in jaw morphology of needlefishes (Teleostei: Belonidae). Syst. Zool. 40, 329-354.

Castanet, J., Grandin, A., Abourachid, A. \& Ricqles, A. de (1996). Expression of growth dynamic in the structure of the periosteal bone in the mallard, Anas platyrhynchos. C. R. Acad. Sci. Paris (Vie) 319, 301-308.

Cracraft, J. (2001). Avian evolution, Gondwana biogeography and the Cretaceous-Tertiary mass extinction event. Proc. R. Soc. Lond. B 268, 459-469.

Cubo, J. (2000). Process heterochronies in endochondral ossification. J. theor. Biol. 205, 343-353, doi:10.1006/ jtbi.2000.2054.

Cubo, J. \& Arthur, W. (2001). Patterns of correlated character evolution in flightless birds: a phylogenetic approach. Evol. Ecol. 14, 693-702. 
CuBo, J. \& CASINos, A. (2000). Incidence and mechanical significance of pneumatization in the long bones of birds. Zool. J. Linn. Soc. 130, 499-510, doi:10.1006/ zjls. 2000.0238 .

Cubo, J., Fouces, V., Gonzalez-Martin, M., Pedroccr, V. \& Rurz, X. (2000). Non-heterochronic developmental changes underlie morphological heterochrony in the evolution of the Ardeidae. J. Evol. Biol. 13, 269-276.

Currey, J. D. \& AleXander, R. McN. (1985). The thickness of the walls of tubular bones. J. Zool. Lond. 206, 453-468.

Eldredge, N. \& Cracraft, J. (1980). Phylogenetic Patterns and the Evolutionary Process. Method and Theory in Comparative Biology. New York: Columbia University Press.

Frorello, C. V. \& German, R. Z. (1997). Heterochrony within species: craniofacial growth in giant, standard, and dwarf rabbits. Evolution 51, 250-261.

Godfrey, L. R. \& Sutherland, M. R. (1995a). Flawed inference: why size-based tests of heterochronic processes do not work. J. theor. Biol. 172, 43-61, doi:10.1006/ jtbi.1995.0004.

Godfrey, L. R. \& SutherLand, M. R. (1995b). What's growth got to do with it? Process and product in the evolution of ontogeny. J. Hum. Evol. 29, 405-431, doi:10.1006/jhev.1995.1066.

Godfrey, L. R. \& Sutherland, M. R. (1996). Paradox of peramorphic peramorphosis: heterochrony and human evolution. Am. J. Phys. Anthr. 99, 17-42.

Goucd, S. J. (1977). Ontogeny and Phylogeny. Cambridge: Belknap Press of Harvard University.

GouLD, S. J. (2000). Of coiled oysters and big brains: how to rescue the terminology of heterochrony, now gone astray. Evol. Dev. 2, 241-248.

Harvey, P. H. \& PAGel, M. (1991). The Comparative Method in Evolutionary Biology. Oxford: Oxford University Press.

KlingenberG, C. P. (1998). Heterochrony and allometry: the analysis of evolutionary change in ontogeny. Biol. Rev. 73, 79-123.

LAIRD, A. K. (1965). Dynamics of relative growth. Growth 29, 249-263.

LAIRD, A. K. (1966). Dynamics of embryonic growth. Growth 30, 263-275.

Laird, A. K. Tyler, S. A. \& Barton, A. D. (1965). Dynamics of normal growth. Growth 29, 233-248.

Legendre, P., Lapornte, F.-J. \& Casgrain, P. (1994). Modeling brain evolution from behavior: a permutational regression approach. Evolution 48, 1487-1499.

MADDIson, W. P. (1991). Squared-change parsimony reconstructions of ancestral states for continuous-valued characters on a phylogenetic tree. Syst. Zool. 40, 304-314.

MaunZ, M. \& German, R. Z. (1997). Ontogeny and limb bone scaling in two New World Marsupials, Monodelphis domestica and Didelphis virginiana. J. Morphol. 231, $117-130$.

McCracken, K. G., Harshman, J., McClellan, D. A. \& Afton, A. D. (1999). Data set incongruence and correlated character evolution: an example of functional convergence in the hind-limbs of stifftail diving ducks. Syst. Biol. 48, 683-714.

PAGEL, M. (1997). Inferring the historical patterns of biological evolution. Nature 401, 877-884.
PAGEL, M. (1999). Inferring evolutionary processes from phylogenies. Zool. Scr. 26, 331-348.

ReIlly, S. M., Wiley, E. O. \& MeInhardT, D. J. (1997). An integrative approach to heterochrony: the distinction between interspecific and intraspecific phenomena. Biol. J. Linn. Soc. 60, 119-143, doi:10.1006/ bijl.1996.0092.

RICKLEFS, R. E. (1973). Patterns of growth in birds. II. Growth rate and mode of development. Ibis $\mathbf{1 1 5}$, 177-201.

Ricklefs, R. E. \& Marks, H. L. (1985). Anatomical response to selection for four-week body mass in japanese quail. Auk 102, 323-333.

Ricqles, S. A. de, Padian, K., Horner, J. R. \& FranCILlon-Vieillot, H. (2000). Palaeohistology of the bones of pterosaurs (Reptilia: Archosauria): anatomy, ontogeny, and biomechanical implications. Zool. J. Linn. Soc. 129, 349-385, doi:10.1006/zjls.1999.0239.

TonNA, E. A. (1961). The cellular complement of the skeletal system studied autoradiographically with tritiated thymidine ( $\left.\mathrm{H}^{3} \mathrm{TDR}\right)$ during growth and aging. J. Biophys. Biochem. Cytol. 9, 813-822.

van Turnen, M. \& Hedges, S. B. (2001). Calibration of avian molecular clocks. Mol. Biol. Evol. 18, 206-213.

\section{Appendix A}

\section{Deduction of the Timing Parameter $\boldsymbol{t}_{\boldsymbol{m}}$}

To calculate the timing parameter $t_{m}$, we shall consider the first derivative of $\sigma(t)$ and calculate its zeros, that is,

$$
\sigma^{\prime}(t)=\sigma(t)\left\{\left[l_{l} \mathrm{e}^{\left(-k_{l} t\right)}\right]-\left[l_{d} \mathrm{e}^{\left(-k_{d} t\right)}\right]\right\} .
$$

Since $\sigma^{\prime}(t)$ is a product of two functions and the first function, $\sigma(t)$, is strictly positive for all $t$, it is quite clear that $\sigma^{\prime}(t)=0$ if and only if the second of these functions vanishes. That is to say, $\sigma^{\prime}(t)=0$ if and only if

$$
\left[l_{l} \mathrm{e}^{\left(-k_{l} t\right)}\right]-\left[l_{d} \mathrm{e}^{\left(-k_{d} t\right)}\right]=0,
$$

which is equivalent to

$$
l_{l} \mathrm{e}^{\left(-k_{l} t\right)}=I_{d} \mathrm{e}^{\left(-k_{d} t\right)}
$$

or

$$
l_{d} / l_{l}=\mathrm{e}^{\left(k_{d}-k_{l}\right)} t
$$

By taking logarithms on both sides, this expression yields

$$
\log l_{d}-\log l_{l}=\left(k_{d}-k_{l}\right) t
$$


that is

$$
t=\left[\log \left(l_{d} / l_{l}\right)\right] /\left(k_{d}-k_{l}\right) .
$$

Note that, since $k_{l}>k_{d}$, and $I_{l}>I_{d}$, this number $t=t_{m}$ is well defined and positive. Therefore, the function $\sigma(t)$ has a unique critical point. Moreover, it is immediately checked that $\sigma^{\prime}(t)<0$ if $t$ is large enough, while the first derivative at hatching $\left[\sigma^{\prime}\left(t_{0}\right)>0\right]$. Since $t_{m}$ is the only point at which $\sigma^{\prime}(t)$ vanishes, this implies that $\sigma(t)$ attains its maximum at the point $t_{m}$ :

$$
t_{m}=\left[\log \left(l_{d} / l_{l}\right)\right] /\left(k_{d}-k_{l}\right)
$$

\section{Appendix B}

\section{Deduction of the Rate Parameter $\beta$}

For any $t$, the function $\sigma^{\prime}(t)$ provides the slope of $\sigma$ at $t$. Therefore, in order to calculate the time $t_{\beta}$ at which the maximum slope $\beta$ is attained, we must find the first zero of the second derivative of $\sigma(t)$, that is

$$
\begin{aligned}
\sigma^{\prime \prime}(t)= & \sigma^{\prime}(t)\left\{\left[l_{l} \mathrm{e}^{\left(-k_{l} t\right)}\right]-\left[l_{d} \mathrm{e}^{\left(-k_{d} t\right)}\right]\right\} \\
& +\sigma(t)\left\{\left[l_{d} k_{d} \mathrm{e}^{\left(-k_{d} t\right)}\right]-\left[l_{l} k_{l} \mathrm{e}^{\left(-k_{l} t\right)}\right]\right\}
\end{aligned}
$$

By substituting in this equality the expression for $\sigma^{\prime}(t)$ calculated above, we get

$$
\begin{aligned}
\sigma^{\prime \prime}(t)= & \sigma(t)\left\{\left[l_{l} \mathrm{e}^{\left(-k_{l} t\right)}\right]-\left[l_{d} \mathrm{e}^{\left(-k_{d} t\right)}\right]\right\}^{2+\sigma^{\prime} t} \\
& \left\{\left[l_{d} k_{d} \mathrm{e}^{\left(-k_{d} t\right)}\right]-\left[l_{l} k_{l} \mathrm{e}^{\left(-k_{l} t\right)}\right]\right\}
\end{aligned}
$$

that is

$$
\begin{aligned}
\sigma^{\prime \prime}(t)= & \sigma(t)\left\{\left[l_{l}^{2} \mathrm{e}^{\left(-2 k_{l} t\right)}\right]+\left[l_{d}^{2} \mathrm{e}^{\left(-2 k_{d} t\right)}\right]\right. \\
& -\left[2 l_{l} l_{d} \mathrm{e}^{-\left(k_{l}+k_{d}\right) t}\right] \\
& \left.+\left[l_{d} k_{d} \mathrm{e}^{\left(-k_{d} t\right)}\right]-\left[l_{l} k_{l} \mathrm{e}^{\left(-k_{l} t\right)}\right]\right\} .
\end{aligned}
$$

We shall denote

$$
\begin{aligned}
\varphi(t)= & \left\{\left[l_{l}^{2} \mathrm{e}^{\left(-2 k_{l} t\right)}\right]+\left[l_{d}^{2} \mathrm{e}^{\left(-2 k_{d} t\right)}\right]\right. \\
& -\left[2 l_{l} l_{d} \mathrm{e}^{-\left(k_{l}+k_{d}\right) t}\right]+\left[l_{d} k_{d} \mathrm{e}^{\left(-k_{d} t\right)}\right] \\
& \left.-\left[l_{l} k_{l} \mathrm{e}^{\left(-k_{l} t\right)}\right]\right\},
\end{aligned}
$$

so that

$$
\sigma^{\prime \prime}(t)=\sigma(t) \varphi(t)
$$

Since the function $\sigma(t)$ is strictly positive, we have that $\sigma^{\prime \prime}(t)=0$ if and only if $\varphi(t)=0$. Therefore, the parameter $t_{\beta}$, the time at maximum slope (maximum rate of ontogenetic change in shape) can be found by solving the equation:

$$
\varphi(t)=0 .
$$

Unfortunately, it is a difficult task to find a general expression for the solutions of this equation, but for any particular empirical values of the parameters $I_{l}, I_{d}, k_{l}, k_{d}$, it is not difficult to approximate the zeros of $\varphi(t)$ by using a standard computer program. Figure 6 provides a particular example: the calculation of the time at maximum slope $\left(t_{\beta}\right)$ in the radius of mallard. Once the first zero $\left(t_{\beta}\right)$ of $\varphi(t)$ has been calculated we can find the maximum slope $\beta$ simply by substituting $t=t_{\beta}$ into the expression for $\sigma^{\prime}(t)$ above [eqn (9)]. That is

$$
\beta=\sigma^{\prime}\left(t_{\beta}\right) .
$$

\section{Appendix C}

Order Anseriformes

\section{List of the Species Studied}

Alopochen aegyptiaca

Anas platyrhynchos

Anser anser

Branta leucopsis

Bucephala clangula

Cairina mostacha

Chloephaga picta

Cygnus cygnus

Cygnus olor

Pleptropterus gambensis

Somateria mollissima

Tachyeres pteneres

Tadorna tadorna

Order Charadriiformes

Larus argentatus

Tringa erythropus

Order Galliformes

Alectoris rufa 
Coturnix coturnix

Phasianus colchicus

Order Palaeognathiformes

Casuarius casuarius

Casuarius unappendiculatus

Dromaius novaehollandidae

Pterocnemia pennata

Rhea americana

Struthio camelus
Order Passeriformes

Corvus corone

Order Sphenisciformes
Aptenodytes patagonicus
Eudyptes chrysocome
Eudyptes chrysolophus
Pygoscelis papua
Spheniscus magellanicus 